\title{
The nitrogen and oxygen abundances in the neutral gas at high redshift ${ }^{\star}$
}

\author{
P. Petitjean ${ }^{1}$, C. Ledoux ${ }^{2}$, and R. Srianand ${ }^{3}$ \\ 1 Institut d'Astrophysique de Paris, CNRS and UPMC Paris 6, UMR7095, 98bis boulevard Arago, 75014 Paris, France \\ e-mail: petitjean@iap.fr \\ 2 European Southern Observatory, Alonso de Córdova 3107, Casilla 19001, Vitacura, Santiago, Chile \\ 3 IUCAA, Post Bag 4, Ganesh Khind, Pune 411 007, India
}

Received 4 September 2007 / Accepted 18 December 2007

\begin{abstract}
Aims. We study the oxygen and nitrogen abundances in the interstellar medium of high-redshift galaxies.

Methods. We use high resolution and high signal-to-noise ratio spectra of damped Lyman- $\alpha$ (DLA) systems detected along the lineof-sight to quasars to derive robust abundance measurements from unsaturated metal absorption lines.

Results. We present results for a sample of 16 high-redshift DLAs and strong sub-DLAs $\left(\log N(\mathrm{H} \mathrm{I})>19.5,2.4<z_{\mathrm{abs}}<3.6\right)$ including 13 new measurements. We find that the oxygen to iron abundance ratio is pretty much constant with $[\mathrm{O} / \mathrm{Fe}] \sim+0.32 \pm 0.10$ for $-2.5<[\mathrm{O} / \mathrm{H}]<-1.0$ with a small scatter around this value. The oxygen abundance follows quite well the silicon abundance within $\sim 0.2$ dex, although the silicon abundance could be slightly smaller for $[\mathrm{O} / \mathrm{H}]<-2$. The distribution of the $[\mathrm{N} / \mathrm{O}]$ abundance ratio, measured from components that are detected in both species, is somehow double peaked: five systems have [N/O] $>-1$ and nine systems have $[\mathrm{N} / \mathrm{O}]<-1.15$. In the diagram $[\mathrm{N} / \mathrm{O}]$ versus $[\mathrm{O} / \mathrm{H}]$, a loose plateau is possibly present at $[\mathrm{N} / \mathrm{O}] \sim-0.9$, which is below the so-called primary plateau as seen in local metal-poor dwarf galaxies ([N/O ] in the range -0.57 to -0.74$)$. No system is seen above this primary plateau whereas the majority of the systems lie well below with a large scatter. All this suggests a picture in which DLAs undergo successive star-bursts. During such an episode, the [N/O] ratio decreases sharply because of the rapid release of oxygen by massive stars, whereas inbetween two bursts, nitrogen is released by low and intermediate-mass stars with a delay and the [N/O] ratio increases.
\end{abstract}

Key words. ISM: abundances - Galaxy: abundances - galaxies: quasars: absorption lines

\section{Introduction}

The production of nitrogen in stars is the subject of strong interest as it is difficult to explain consistently the nitrogen abundances measured in different astrophysical environments. New abundance measurements in the interstellar medium (ISM) of our Galaxy, as well as in external galaxies, are of prime importance in these discussions. This could help decide what the sites of the nitrogen production are. It is generally believed that the main contributors are the long-lived intermediate mass stars that are progenitors of asymptotic giant branch (AGB) stars. The contribution from massive stars is still uncertain.

The main nucleosynthetic pathway for the production of nitrogen is the CNO cycle, which takes place in the stellar $\mathrm{H}$ burning layers. Nitrogen is thought to have both primary and secondary origins depending on whether the seed carbon and oxygen nuclei are produced by the star itself (primary) or are already present in the ISM from which the star forms, in which case, carbon and oxygen seeds are left-overs from previous generations of stars (secondary). Secondary production can happen in the H-burning layers of all stars as the carbon seed is present,

\footnotetext{
* Based on observations carried out at the European Southern Observatory (ESO), under visitor mode progs. ID 65.O-0063, 66.A0624, 67.A-0078 and 68.A-0600 with the UVES echelle spectrograph installed at the ESO Very Large Telescope (VLT), unit Kueyen, on mount Paranal in Chile. Also based on archival data from progs. 68.A0492 (PI: D’Odorico), 68.B-0115 (PI: Molaro) and 69.A-0051 (PI: Pettini).
}

by definition, in these layers. Primary nitrogen is produced when carbon, synthesized in the helium-burning shell of the star, penetrates into the hydrogen-burning upper shell, where it is transformed into nitrogen by the $\mathrm{CNO}$ cycle. This happens in intermediate mass stars $\left(4 \leq M / M_{\odot} \leq 7\right)$ during the AGB phase (Henry et al. 2000). Large uncertainties affect the theoretical predictions of both the primary and secondary nucleosynthesis of nitrogen in low and intermediate-mass stars and the possible contribution of primary nitrogen from massive stars (Woosley \& Weaver 1995; Marigo 2001; Maeder \& Meynet 2002).

It is believed that the determination of the nitrogen abundance in objects with metallicities spread over a large range may help to answer this question. Therefore, it is usual to derive metallicities in nearby low-metallicity emission line galaxies (e.g. Nava et al. 2006; Izotov et al. 2006) or low-metallicity stars in our Galaxy (Spite et al. 2005). A complementary approach is to derive metallicities directly at high redshift in damped Lyman- $\alpha$ (DLA) systems (Pettini et al. 2002; Prochaska et al. 2002; Centurión et al. 2003) that have metallicities typically in the range $-2.5 \leq Z / Z_{\odot} \leq-1$. Due to the large $\mathrm{H}$ I column densities $\left(\log N(\mathrm{H} \mathrm{I}) \geq 10^{20} \mathrm{~cm}^{-2}\right)$ and the conspicuous presence of metals, DLAs are believed to arise in high-redshift galaxies or at least to be located close to regions where star-formation occurs.

It is usual to discuss these issues using the diagram giving the nitrogen to oxygen abundance ratio versus the oxygen abundance. In the case of secondary production, the ratio of nitrogen to oxygen abundances increases with increasing oxygen metallicity, whereas for primary production, the ratio remains constant 
as nitrogen tracks oxygen. In $\mathrm{H}$ II regions of nearby galaxies, a trend in the $[\mathrm{N} / \mathrm{O}]$ abundance ratio is seen with a primary plateau at low oxygen abundances and a secondary behavior for abundances $[\mathrm{O} / \mathrm{H}]>-1$ (van Zee et al. 1998; Izotov \& Thuan 1999). DLA systems have a very different behavior in this diagram with $[\mathrm{N} / \alpha]$ (see below) measurements well below the primary plateau (Pettini et al. 2002; Centurión et al. 2003). This is probably related to the star-formation history of these objects.

Oxygen and nitrogen abundances can be derived directly from the $N(\mathrm{O}$ I $) / N(\mathrm{H} \mathrm{I})$ and $N(\mathrm{~N} \mathrm{I}) / N(\mathrm{H} \mathrm{I})$ column density ratios. Because of efficient charge exchange reactions that link the neutral species together, the ionization corrections are negligible for $\log N(\mathrm{H} \mathrm{I})>19.5$ (Viegas 1995). Accurate O I and N I column densities are, however, difficult to derive. The main reason is that the absorption lines are located in the Lyman- $\alpha$ forest and are often blended. In addition, and this is especially true for $\mathrm{O}$ I, absorption lines are easily saturated. For $\mathrm{N}$ I the situation is less dramatic because the nitrogen abundance is smaller and N I has two triplets around $\lambda 1134$ and $\lambda 1200 \AA$. On the contrary, the main $\mathrm{O}$ I absorption feature at $\lambda 1302$ is a single line and is often saturated. Other weaker absorption lines are found much further in the blue, therefore deep in the Lyman- $\alpha$ forest and in a wavelength range where good a signal-to-noise ratio (SNR) is difficult to obtain. This is why, in previous studies, the oxygen abundance has often been replaced by that of another $\alpha$-element, such as sulfur or silicon (Pettini et al. 2002; Centurión et al. 2003; Henry \& Prochaska 2007). Using silicon instead of oxygen, Centurión et al. (2003) claimed that $75 \%$ of DLA systems show a mean value $[\mathrm{N} / \mathrm{Si}]=-0.87$ with 0.17 dex dispersion, corresponding approximately to the primary plateau observed locally, and $25 \%$ are clustered around $[\mathrm{N} / \mathrm{Si}]=-1.5$ with even less dispersion $(0.05 \mathrm{dex})$, the transition between low and high [N/Si] values happening at $[\mathrm{N} / \mathrm{H}]=-2.8$ (note that the latter transition may occur at $[\mathrm{N} / \mathrm{H}]=-3$, see Molaro et al. 2003).

Here we present the $(\mathrm{N} / \mathrm{O})$ vs. $(\mathrm{O} / \mathrm{H})$ diagram using robust determinations of oxygen and nitrogen metallicities derived from unsaturated absorption lines selected from the largest UVES sample of DLA systems available up to now (Ledoux et al. 2003, 2006a). In Sect. 2 we present our sample. We describe the $\mathrm{O}$ and $\mathrm{N}$ abundance measurements in Sect. 3 and discuss the results and implications in Sects. 4 and 5.

\section{UVES DLA sample}

Most of the systems in our sample were selected from the followup of the Large Bright QSO Survey (Wolfe et al. 1995) and observed at the VLT with UVES between 2000 and 2004 in the course of a systematic search for molecular hydrogen at $z_{\text {abs }}>1.7$ (Petitjean et al. 2000; Ledoux et al. 2003). Our sample comprises 61 bona-fide DLA systems $(\log N(\mathrm{HI}) \geq 20.3)$ and 13 strong sub-DLA systems with total neutral hydrogen column densities in the range $19.5 \lesssim \log N(\mathrm{HI})<20.3$. Characteristics of the systems (metallicities, N(H I) column densities, kinematics), except for four sub-DLAs with $\log N(\mathrm{H} \mathrm{I})=19.7-19.8$ ), are given in Ledoux et al. (2006a). The absorption line analysis was performed in a homogeneous manner using standard Voigt-profile fitting techniques adopting the oscillator strengths compiled by Morton (2003). Total column densities were derived as the sum of the column densities measured in individual components of the line profiles. Average gas phase metallicities relative to solar, $[\mathrm{X} / \mathrm{H}] \equiv \log [N(\mathrm{X}) / N(\mathrm{H})]-\log [N(\mathrm{X}) / N(\mathrm{H})]_{\odot}$, were calculated using solar abundances from Lodders (2003).

As we are interested in measuring the $[\mathrm{O} / \mathrm{H}]$ and $[\mathrm{N} / \mathrm{H}]$ abundances from the $N(\mathrm{O} \mathrm{I}), N(\mathrm{~N} \mathrm{I})$ and $N(\mathrm{H} \mathrm{I})$ column densities, we have to select systems where we can derive accurate column densities, e.g., where at least one transition from both $\mathrm{N}$ I and $\mathrm{O} \mathrm{I}$ is not strongly saturated. Note that the transitions from these elements are mostly redshifted in the Lyman- $\alpha$ forest and we require in addition that at least one unsaturated transition be free of blending. From the above sample and under these conditions, we selected 13 sytems where we could measure $N(\mathrm{O} \mathrm{I})$ and $N(\mathrm{~N}$ I $)$ column densities accurately.

From the literature, we added three measurements at $z_{\mathrm{abs}}=3.390,2.844$ and 3 toward, respectively, Q 0000-263 (Molaro et al. 2001), Q 1946+769 and QXO 0001 (Prochaska et al. 2002). As a consequence of our selection criteria, we did not include the system at $z_{\mathrm{abs}}=4.466$ toward Q 0307-4945 (Dessauges-Zavadsky et al. 2001) as the complex O I and N I velocity profiles, spread over more than $200 \mathrm{~km} \mathrm{~s}^{-1}$, are highly saturated and/or blended. Neither did we include the systems at $z_{\mathrm{abs}}=2.076$ and 2.456 toward, respectively, Q 2206-199 and Q 1409+095 (Pettini et al. 2002) because the oxygen abundance can only be ill-defined from strongly saturated O I $\lambda 1302$ absorption lines. In the case of Q 2206-199, the strongest N I feature is also blended (Molaro et al. 2003).

Ionization may be a concern when deriving the (N/O) abundance ratio from the $N(\mathrm{~N} \mathrm{I}) / N(\mathrm{O} \mathrm{I})$ column density ratio (Viegas 1995; Prochaska et al. 2002). If the gas is neutral then charge exchange reactions are fast enough so that $\mathrm{O}$ I and $\mathrm{N}$ I are both tied to $\mathrm{H} \mathrm{I}$ and there is no need for correction for $\log N(\mathrm{H} \mathrm{I})>19.5$ (Viegas 1995). In case of the presence of enough hard photons (or cosmic rays) the ionization balance could be displaced towards higher ionization species and both $\mathrm{O}$ I and $\mathrm{N}$ I could be over-ionized compared to H I. However, for most DLA systems, hard photons come predominantly from the background ionization field. The ionization parameter for this field at $z=3$ is $U<10^{-4}$ for typical densities expected for DLAs $\left(n>1 \mathrm{~cm}^{-3}\right.$; see e.g. Petitjean et al. 1992), and ionization corrections are again small. Indeed, Prochaska et al. (2002) inspected about twenty DLAs and concluded that the ionization correction for $[\mathrm{N} / \alpha]$ is at most of the order of +0.1 dex. This amount should probably be added to the measurement errors. In the following we did not apply any correction as we show that there is no correlation between $[\mathrm{N} / \mathrm{O}]$ and $\log N(\mathrm{H} \mathrm{I})$.

\section{Column densities and abundances}

We performed in a usual manner Voigt profile fitting of absorption lines associated to the 13 systems we selected from our DLA sample (see e.g. Ledoux et al. 2006a; Erni et al. 2006). The overall decomposition in subcomponents is derived from the simultaneous fit of numerous absorption lines from different species (most importantly Zn II, Fe II and Si II) redshifted outside the Lyman- $\alpha$ forest and free from blending. We used the resulting component structure to detect $\mathrm{N}$ I and $\mathrm{O}$ I features and confirm that they are not blended. Column densities obtained for each of the components are given in Table 1. Fits are shown in Figs. 8-10. The fits to the absorption lines in the $z_{\mathrm{abs}}=3.025$ system towards Q 0347-383 can be seen in Ledoux et al. (2003). Errors for individual components are the $1 \sigma$ errors from Voigt profile fitting, as given by FitLyman.

Following the usual procedure, $[\mathrm{N} / \mathrm{H}]$ and $[\mathrm{O} / \mathrm{H}]$ abundances are obtained by adding the column densities in all detected individual components. For O I, all the systems are dominated by one strong component in which we do not expect much ionization correction (see the next section for details on each of the systems). It is possible that in some of the weak components the gas is partially ionized and that some ionization correction should be 
Table 1. Results of Voigt-profile fitting.

\begin{tabular}{|c|c|c|c|c|c|c|c|c|c|}
\hline Name & $n$ & $z_{\mathrm{abs}}$ & $\begin{array}{r}\log N(\mathrm{H} \mathrm{I}) \\
\left(\mathrm{cm}^{-2}\right)\end{array}$ & $\begin{array}{r}\log N(\mathrm{O} \mathrm{I}) \\
\left(\mathrm{cm}^{-2}\right)\end{array}$ & $\sigma$ & $\overline{[\mathrm{O} / \mathrm{H}]}$ & $\begin{array}{r}\log N(\mathrm{~N} \mathrm{I}) \\
\left(\mathrm{cm}^{-2}\right)\end{array}$ & $\sigma$ & $\overline{[\mathrm{N} / \mathrm{H}]}$ \\
\hline \multirow{5}{*}{ Q 0102-190 } & 1 & 2.92646 & $20.00 \pm 0.10$ & 14.68 & 0.16 & & 12.67 & 0.11 & \\
\hline & 2 & 2.92661 & & 14.66 & 0.17 & & $<12.35$ & $d$ & \\
\hline & 3 & 2.92727 & & 14.20 & 0.02 & & 12.38 & 0.09 & \\
\hline & 4 & 2.92771 & & 14.41 & 0.02 & & 12.55 & 0.06 & \\
\hline & Tot & & & 15.13 & 0.08 & -1.56 & 12.76 & 0.11 & -3.07 \\
\hline \multirow[t]{3}{*}{ Q 0112-306 } & 1 & 2.41844 & $20.50 \pm 0.08$ & 14.78 & 0.12 & & 12.66 & 0.08 & \\
\hline & 2 & 2.41861 & & 14.45 & 0.06 & & 12.99 & 0.04 & \\
\hline & Tot & & & 14.95 & 0.08 & -2.24 & 13.16 & 0.04 & -3.17 \\
\hline \multirow[t]{3}{*}{ Q $0347-383^{a}$} & 1 & 3.02463 & $20.73 \pm 0.05$ & 16.12 & 0.12 & & 14.15 & 0.03 & \\
\hline & 2 & 3.02485 & & 16.18 & 0.18 & & 14.47 & 0.03 & \\
\hline & Tot & & & 16.45 & 0.11 & -0.97 & 14.64 & 0.02 & -1.92 \\
\hline Q $0841+129$ & 1 & 2.47622 & $20.80 \pm 0.10$ & 16.09 & 0.03 & -1.40 & 13.94 & 0.02 & -2.69 \\
\hline \multirow[t]{3}{*}{ Q $0913+072^{b}$} & 1 & 2.61829 & $20.35 \pm 0.10$ & 14.08 & 0.02 & & $<12.10$ & $d$ & \\
\hline & 2 & 2.61844 & & 14.43 & 0.02 & & $<12.20$ & & \\
\hline & Tot & & & 14.59 & 0.02 & -2.45 & $<12.45$ & & $<-3.73$ \\
\hline Q 1108-077 & 1 & 3.60767 & $20.37 \pm 0.07$ & 15.37 & 0.03 & -1.69 & $<12.84$ & $d$ & $<-3.36$ \\
\hline Q $1337+113$ & 1 & 2.50793 & $20.12 \pm 0.05$ & 14.90 & 0.10 & -1.91 & 12.84 & 0.10 & -3.11 \\
\hline \multirow[t]{3}{*}{ Q $1337+113$} & 1 & 2.79557 & $21.00 \pm 0.08$ & 15.21 & 0.12 & & $<12.40$ & $d$ & \\
\hline & 2 & 2.79581 & & 15.59 & 0.08 & & 13.98 & 0.03 & \\
\hline & Tot & & & 15.74 & 0.07 & -1.95 & 13.99 & 0.03 & -2.84 \\
\hline Q 1340-136 & 1 & 3.11835 & $20.05 \pm 0.08$ & 15.52 & 0.02 & -1.22 & 13.28 & 0.02 & -2.60 \\
\hline \multirow[t]{3}{*}{ Q $1409+095^{c}$} & 1 & 2.45595 & $20.53 \pm 0.08$ & 15.05 & 0.02 & & $<13.26$ & d & \\
\hline & 2 & 2.45644 & & 15.00 & 0.02 & & $<13.14$ & & \\
\hline & Tot & & & 15.33 & 0.02 & -1.89 & $<13.51$ & & $<-2.85$ \\
\hline Q $1409+095^{c}$ & 1 & 2.66821 & $19.80 \pm 0.08$ & 15.18 & 0.02 & -1.31 & 13.50 & 0.02 & -2.13 \\
\hline \multirow[t]{4}{*}{ Q 2059-360 } & 1 & 3.08262 & $20.98 \pm 0.08$ & 15.63 & 0.06 & & 13.49 & 0.02 & \\
\hline & 2 & 3.08293 & & 15.85 & 0.05 & & 13.71 & 0.02 & \\
\hline & 3 & 3.08316 & & 15.02 & 0.12 & & 12.85 & 0.14 & \\
\hline & Tot & & & 16.09 & 0.04 & -1.58 & 13.95 & 0.02 & -2.86 \\
\hline \multirow[t]{7}{*}{ Q 2332-094 } & 1 & 3.05633 & $20.50 \pm 0.07$ & 14.97 & 0.02 & & $<12.93$ & $d$ & \\
\hline & 2 & 3.05658 & & 14.46 & 0.03 & & $<12.93$ & $d$ & \\
\hline & 3 & 3.05677 & & 14.71 & 0.07 & & $<12.93$ & $d$ & \\
\hline & 4 & 3.05690 & & 15.32 & 0.02 & & $<12.93$ & $d$ & \\
\hline & 5 & 3.05723 & & 15.67 & 0.04 & & 13.73 & 0.03 & \\
\hline & 6 & 3.05737 & & 14.66 & 0.04 & & $<12.93$ & $d$ & \\
\hline & Tot & & & 15.95 & 0.02 & -1.24 & 13.73 & 0.03 & -2.60 \\
\hline
\end{tabular}

${ }^{a}$ Also Ledoux et al. (2003); ${ }^{b}$ also Erni et al. (2006); ${ }^{c}$ also Pettini et al. (2002); ${ }^{d} 3 \sigma$ detection limit.

applied to these components. However, this ionization correction is probably smaller than 0.2 dex in the weakest components and these components do not account, in total, for more than $\sim 20 \%$ of the total column density. We therefore believe that the error on $[\mathrm{O} / \mathrm{H}]$ due to the procedure is less than 0.2 dex for the whole system (see also the discussion in Prochaska et al. 2002). For $\mathrm{N}$ I, the above error is less as the transitions are weaker and the species are always detected in less components.

For the $[\mathrm{N} / \mathrm{O}]$ ratio, we use the column densities in the components where both species are detected. Note that when $\mathrm{N} \mathrm{I}$ is detected in only one component, this is always the strongest $\mathrm{O}$ I component. It is interesting to note that, where $\mathrm{N}$ I and $\mathrm{O}$ I are detected in several components, as toward Q 2332-094, the $[\mathrm{N} / \mathrm{O}]$ ratios are similar in all components.

We adopt the oxygen and nitrogen solar abundances from Lodders (2003): $12+(\mathrm{O} / \mathrm{H})_{\odot}=8.69$ and $12+(\mathrm{N} / \mathrm{H})_{\odot}=7.83$. Note that the main discussion on the production of these elements is little dependent on the exact adopted values. Depletion onto dust-grains is known to be much smaller in DLA systems compared to the ISM of our Galaxy, mostly because of smaller (by a factor of at least ten) metallicities. Depletions should therefore be much smaller than in the diffuse galactic ISM clouds where it has been shown that, apart from local effects, the [N/O] ratio is similar to the solar ratio (Knauth et al. 2006).

\section{Comments on individual systems}

Q 0000-263: We adopt the column densities from Molaro et al. (2001). The oxygen abundance is derived from unsaturated O I $\lambda \lambda 925,950$ absorption lines. The nitrogen abundance is derived from the unsaturated $\lambda 953$ line and the $\lambda 1134$ triplet. The system is modeled with only one component.

Q 0102-190 (Fig. 8, top panel): The structure of the system is derived from the fit of Fe II and Si II lines. $N(\mathrm{~N}$ I $)$ is derived from a $\lambda 1199$ optically thin feature corresponding in redshift with the strongest O I component. We add all O I column densities (obtained from optically thin $\lambda \lambda 976,1039$ features) to calculate the $\mathrm{O}$ abundance. Note, however, that the red satellites do not contribute much. The $[\mathrm{N} / \mathrm{O}]$ ratio is taken as the column density ratio in the main central component.

Q 0112-306 (Fig. 8): The two-component structure of the system is very well defined from $\mathrm{Si}$ II and Fe II absorption lines. This is why we are confident that the $\log N(\mathrm{O}$ I) column density derived from the moderately saturated $\lambda 1302$ absorption is robust. $\mathrm{N} \mathrm{I}$ is detected through an optically thin $\lambda 1199$ feature. 
Table 2. Metal abundances in Damped Lyman- $\alpha$ systems.

\begin{tabular}{|c|c|c|c|c|c|c|c|c|}
\hline Name & $z_{\mathrm{abs}}$ & {$[\mathrm{Fe} / \mathrm{H}]$} & {$[\mathrm{O} / \mathrm{H}]$} & {$[\mathrm{N} / \mathrm{H}]$} & $(\mathrm{O} / \mathrm{H})+12^{c}$ & $(\mathrm{~N} / \mathrm{O})^{d}$ & {$[\mathrm{~S} / \mathrm{H}]$} & {$[\mathrm{Si} / \mathrm{H}]$} \\
\hline Q $0000-263^{a}$ & 3.390 & $-2.00(0.09)$ & $-1.67(0.13)$ & $-2.50(0.08)$ & 7.02 & $-1.69(0.10)$ & $-1.89(0.09)$ & $-1.88(0.08)$ \\
\hline Q 0102-190 & 2.926 & $-1.68(0.10)$ & $-1.56(0.13)$ & $-3.07(0.15)$ & 7.13 & $-2.30(0.16)$ & $-1.37(0.10)$ & $-1.50(0.10)$ \\
\hline Q 0112-306 & 2.418 & $-2.64(0.09)$ & $-2.24(0.12)$ & $-3.17(0.09)$ & 6.45 & $-1.79(0.09)$ & $-1.25(0.08)$ & $-2.42(0.08)$ \\
\hline Q 0347-383 & 3.025 & $-1.85(0.05)$ & $-0.97(0.12)$ & $-1.92(0.05)$ & 7.72 & $-1.81(0.11)$ & $-1.18(0.06)$ & $-1.49(0.06)$ \\
\hline Q $0841+129$ & 2.476 & $-1.79(0.10)$ & $-1.40(0.10)$ & $-2.69(0.10)$ & 7.29 & $-2.15(0.04)$ & $-1.55(0.10)$ & $-1.58(0.10)$ \\
\hline Q $0913+072$ & 2.618 & $-2.72(0.10)$ & $-2.45(0.10)$ & $<-3.73$ & 6.24 & $<-2.14$ & $-1.66(0.10)$ & $-2.57(0.10)$ \\
\hline Q 1108-077 & 3.608 & $-1.96(0.07)$ & $-1.69(0.08)$ & $<-3.36$ & 7.00 & $<-2.53$ & & $-1.57(0.07)$ \\
\hline Q $1337+113$ & 2.508 & $-2.23(0.05)$ & $-1.91(0.11)$ & $-3.11(0.11)$ & 6.78 & $-2.06(0.14)$ & & $-1.84(0.06)$ \\
\hline Q $1337+113$ & 2.796 & $-2.14(0.08)$ & $-1.95(0.10)$ & $-2.84(0.09)$ & 6.74 & $-1.61(0.09)$ & & $-1.84(0.09)$ \\
\hline Q 1340-136 & 3.118 & $-1.59(0.08)$ & $-1.22(0.08)$ & $-2.60(0.08)$ & 7.47 & $-2.24(0.03)$ & $-1.41(0.08)$ & $-1.18(0.08)$ \\
\hline Q 1409+095 & 2.456 & $-2.43(0.08)$ & $-1.89(0.08)$ & $<-2.85$ & 6.80 & $<-1.82$ & $-1.74(0.08)$ & $-2.17(0.08)$ \\
\hline Q 1409+095 & 2.668 & $-1.51(0.08)$ & $-1.31(0.08)$ & $-2.13(0.08)$ & 7.38 & $-1.68(0.03)$ & $-1.40(0.09)$ & $-1.28(0.08)$ \\
\hline Q $1946+769^{b}$ & 2.844 & $-2.50(0.06)$ & $-2.14(0.06)$ & $-3.51(0.07)$ & 6.55 & $-2.23(0.04)$ & & $-2.21(0.06)$ \\
\hline Q 2059-360 & 3.083 & $-1.97(0.08)$ & $-1.58(0.09)$ & $-2.86(0.08)$ & 7.11 & $-2.14(0.04)$ & $-1.76(0.09)$ & $-1.66(0.09)$ \\
\hline Q 2332-094 & 3.057 & $-1.60(0.07)$ & $-1.24(0.07)$ & $-2.60(0.08)$ & 7.45 & $-1.94(0.05)$ & $-1.32(0.08)$ & \\
\hline $\mathrm{QXO} 0001^{b}$ & 3 & $<-1.08$ & $-1.62(0.05)$ & $-3.22(0.06)$ & 7.07 & $-2.46(0.05)$ & & $-1.79(0.05)$ \\
\hline
\end{tabular}

${ }^{a}$ Molaro et al. (2001); ${ }^{b}$ Prochaska et al. (2002); ${ }^{c} \sigma=\sigma([\mathrm{O} / \mathrm{H}]) ;{ }^{d}$ errors are taken as square root of the geometrical mean of the variances of $N(\mathrm{~N}$ I $)$ and $N(\mathrm{O} \mathrm{I})$, as given in Table 1, except for Q 0102-190, Q 1337+113 at $z_{\text {abs }}=2.796$ and Q 2332-094 for which we consider only the main components of the systems to calculate the $[\mathrm{N} / \mathrm{O}]$ ratio (see text in Sect. 3).

Q 0347-383: This system has been studied by Levshakov et al. (2002) and Prochaska et al. (2002). The UVES data have also been reanalyzed by Ledoux et al. (2003). $N(\mathrm{~N}$ I) is constrained by optically thin $\lambda 1134$ features and $N(\mathrm{O}$ I $)$ by $\lambda 950$ and $\lambda 974$. Note that Levshakov et al. (2002) derived $\log N(\mathrm{~N}$ I $)=14.89$, 0.25 dex larger than our result. We believe this is because the latter authors do not restrict their fit to optically thin lines.

Q $0841+129$ (Fig. 8): This is a beautiful one-component system with several optically thin N I components (see also Centurión et al. 2002). N(O I) is derived from the consistent fit of optically thick $\lambda 1302$ and 1039 features and the moderately saturated $\lambda 950$ absorption. Centurión et al. (2003) found a $N$ I column density 0.16 dex larger. This is likely due to the difference in data quality.

Q $0913+072$ (Fig. 8, bottom panel): The absorption profile is made of two closely blended components of similar strength. An upper limit on $\log N(\mathrm{~N} \mathrm{I}$ ) is obtained from $\lambda 1199$ (a feature is present but below the $3 \sigma$ detection limit, see also Erni et al. 2006). Upper limits are derived from the noise in the adjacent continuum; when two components are present we conservatively consider each component independently. $N(\mathrm{O} \mathrm{I})$ is derived from optically thin $\lambda 1039$.

Q 1108-077 (Fig. 9, top panel): This is a one-component system. An upper limit on $N(\mathrm{~N} \mathrm{I})$ is obtained from $\lambda 1134 . N(\mathrm{O} \mathrm{I})$ is derived from an optically thin $\lambda 1039$ feature.

Q $1337+113, z_{\text {abs }}=2.508$ (Fig. 9): This is again a single component system with well constrained $\mathrm{N}$ I and O I column densities from optically thin, respectively, $\lambda 1200$ and $\lambda 1039$ absorption lines.

Q $1337+113, z_{\mathrm{abs}}=2.796$ (Fig. 9): There are two components in this system. $N(\mathrm{~N} \mathrm{I})$ and $N(\mathrm{O}$ I) are both well defined from optically thin, respectively, $\lambda 1134,1200$ and $\lambda 976$ features. The component at $z_{\mathrm{abs}}=2.79581$ is the strongest and is the only component detected in $\mathrm{N}$ I. It is possible that $\mathrm{N}$ I is somewhat ionized in the component at $z_{\text {abs }}=2.79557$. As done for all systems, we add the $\mathrm{O}$ I column densities to calculate the oxygen abundance but take the N/O ratio from the component at $z_{\mathrm{abs}}=2.79581$.

Q 1340-136 (Fig. 9, bottom panel): This is a beautiful onecomponent system with optically thin transitions.

Q $1409+095, z_{\text {abs }}=2.456$ (Fig. 10 , top panel): Although there may be some absorption at the wavelengths corresponding to the
$\mathrm{N}$ I transitions, we derive only an upper limit on $N(\mathrm{~N}$ I) in this two-component system (see also Pettini et al. 2002). The O I column density is well defined by optically thin $\lambda 1039$ absorption. Q 1409+095, $z_{\mathrm{abs}}=2.668$ (Fig. 10): The consistency of the $\lambda \lambda 1199,1200$ and $\lambda 1134$ features makes us believe that $\mathrm{N} \mathbf{I}$ is present in this one-component system. The $\mathrm{O}$ I column density is well defined by optically thin $\lambda 1039$ and $\lambda 950$ absorptions. Q 1946+769: We use the measurements by Prochaska et al. (2002).

Q 2059-360 (Fig. 10): N I and O I are detected in the three components of this system in, respectively, optically thin $\lambda 1134,1200$ and $\lambda 950,976$ transitions. It is important to note that the [N/O] ratio is similar for the three components. This supports the assumption made in this paper that ionization corrections are negligible.

Q 2332-094 (Fig. 10, bottom panel): The system is complex but dominated by two main components. We add all O I column densities but the contribution of the three satellite components is negligible. $\mathrm{N}$ I is detected in a single component corresponding to the strongest $\mathrm{O}$ I component at $z_{\mathrm{abs}}=3.05723$ and we use the $[\mathrm{N} / \mathrm{O}]$ ratio in this component.

QXO 0001: We use the measurements by Prochaska et al. (2002). Note that only one digit is given by these authors for the absorption redshift.

\section{Results}

A summary of the abundances of interest here, measured in the sixteen systems, is given in Table 2.

\subsection{The oxygen abundance in DLAs}

Few oxygen abundance measurements have been reported up to now in DLAs (see Molaro et al. 2000; Pettini et al. 2002). The main reason is that most of the time O I $\lambda 1302$ absorption lines are badly blended or saturated. High resolution and high SNR spectra covering the bluer part of the optical wavelength range are needed to detect weaker O I transitions. Thanks to the large number of DLAs observed in the course of the survey for molecular hydrogen and to the UVES sensitivity into the blue, we could build a sample of robust measurements. 


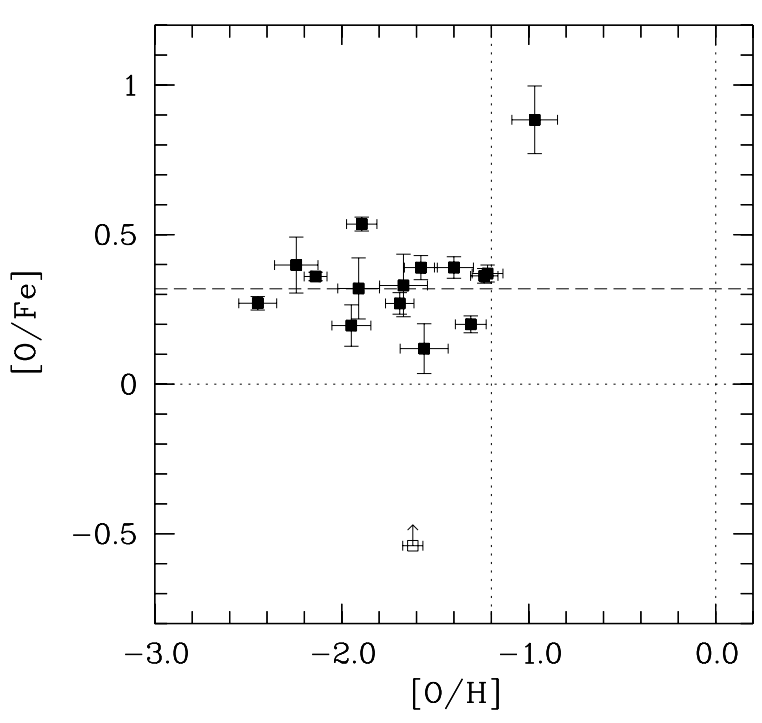

Fig. 1. $[\mathrm{O} / \mathrm{Fe}]$ abundance ratio versus oxygen abundance $[\mathrm{O} / \mathrm{H}]$. The dashed and dotted lines are drawn for convenience.

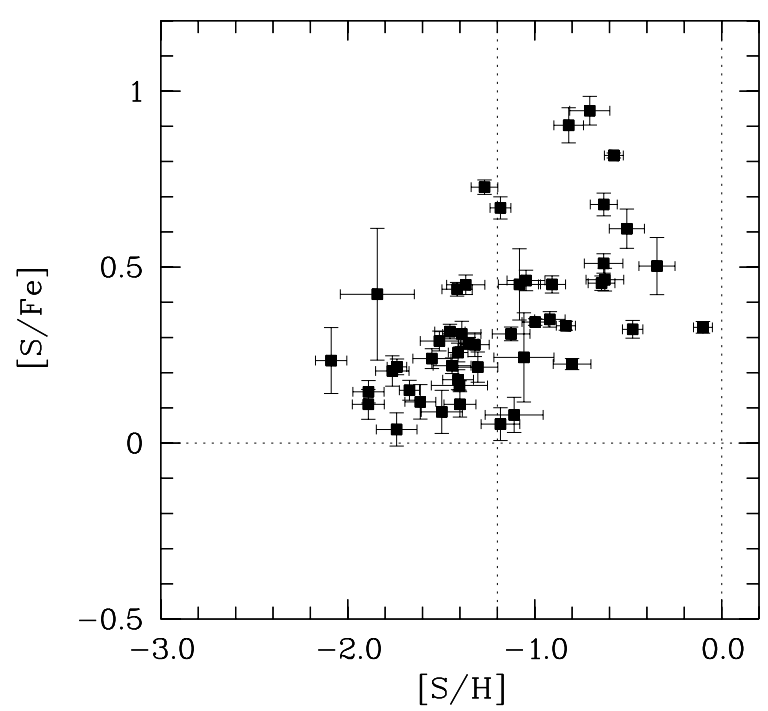

Fig. 2. Sulfur to iron abundance ratio, $[\mathrm{S} / \mathrm{Fe}]$, versus sulfur abundance relative to solar, $[\mathrm{S} / \mathrm{H}]$. The lines are drawn for convenience.

Figure 1 gives the $[\mathrm{O} / \mathrm{Fe}]$ abundance ratio versus the oxygen abundance. In principle, this ratio should be corrected for depletion of metals onto dust-grains. However, this correction is known to be negligible for $[\mathrm{X} / \mathrm{H}]<-1.2$ (Prochaska \& Wolfe 2003; Ledoux et al. 2003; Wolfe et al. 2005). To support this idea, we plot in Fig. 2 the abundance ratio [S/Fe] versus the sulfur abundance $[\mathrm{S} / \mathrm{H}]$ for all systems in our DLA sample (Ledoux et al. 2006a). Sulfur and iron are known to be, respectively, little and strongly depleted onto dust-grains. It is apparent that the scatter of the $[\mathrm{S} / \mathrm{Fe}]$ values is much less below $[\mathrm{S} / \mathrm{H}]=-1.2$ than above. This behavior is a consequence of the largest depletion of iron onto dust-grains for $[\mathrm{S} / \mathrm{H}]>-1.2$.

It can be seen that because of our choice to reject the systems where all O I transitions are badly saturated, our sample is biased against metallicities $[\mathrm{O} / \mathrm{H}]>-1$. All our systems except one have metallicities in the range $-2.5<[\mathrm{O} / \mathrm{H}]<-1.2$. The stricking result here is that the $[\mathrm{O} / \mathrm{Fe}]$ ratio is pretty constant

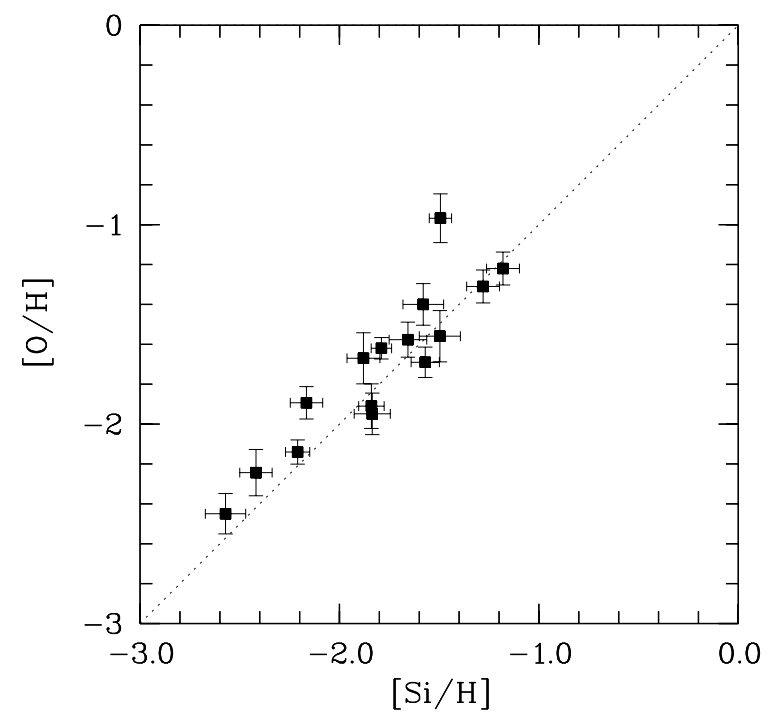

Fig. 3. Oxygen abundance relative to solar, $[\mathrm{O} / \mathrm{H}]$, versus silicon abundance $[\mathrm{Si} / \mathrm{H}]$. Dotted line is for $[\mathrm{O} / \mathrm{H}]=[\mathrm{Si} / \mathrm{H}]$.

over the range studied. The mean value of the 14 measurements at $[\mathrm{O} / \mathrm{H}]<-1$ (therefore excluding $\mathrm{Q}$ 0347-383) is $[\mathrm{O} / \mathrm{Fe}]=0.32 \pm 0.10$ with a slight tendency of this ratio to increase at a lower metallicity. The only departure from this picture is the point at $[\mathrm{O} / \mathrm{H}]=-0.97,[\mathrm{O} / \mathrm{Fe}]=0.88$, from the system at $z_{\mathrm{abs}}=3.025$ towards Q $0347-383$. This is probably due to depletion onto dust-grains $([\mathrm{Zn} / \mathrm{Fe}]=0.72)$. Actually, molecular hydrogen, although with very low molecular fraction, is detected in this system (Levshakov et al. 2002; Ledoux et al. 2003), supporting the presence of dust.

As the oxygen abundance is difficult to measure, the Si abundance is often used instead. Prochaska \& Wolfe (2002) and Dessauges-Zavadsky et al. (2007) have found that the [Si/Fe] ratio is pretty constant amongst DLA systems around a value of +0.43 with a relatively small dispersion. We plot in Fig. 3 the $[\mathrm{O} / \mathrm{H}]$ abundance versus the $[\mathrm{Si} / \mathrm{H}]$ abundance. It is apparent that the two abundances correlate well. There may be a slight tendency for oxygen to be more abundant than Silicon for $[\mathrm{O} / \mathrm{H}]<-2$ but there are only four measurements there.

\subsection{The $[N / O]$ abundance ratio}

Since we will use two different notations, we would first like to clarify them. The (N/O) abundance ratio is simply the ratio of the nitrogen to oxygen abundances, $(\mathrm{O} / \mathrm{H})=\log N(\mathrm{O} \mathrm{I})-\log N(\mathrm{H} \mathrm{I})$ and the same for $(\mathrm{N} / \mathrm{H})$. This ratio is often plotted versus the oxygen abundance written as $(\mathrm{O} / \mathrm{H})+12$. It is, however, very convenient, and usual, to refer to solar abundances, $[\mathrm{O} / \mathrm{H}]=(\mathrm{O} / \mathrm{H})-(\mathrm{O} / \mathrm{H})_{\odot}$. In that case, solar abundances should be specified. We use: $12+(\mathrm{O} / \mathrm{H})_{\odot}=8.69$ and $12+(\mathrm{N} / \mathrm{H})_{\odot}=7.83$. Therefore, $[\mathrm{N} / \mathrm{O}]=(\mathrm{N} / \mathrm{O})+0.86$. What is most important in the following is the value assigned to the so-called "primary plateau". Nava et al. (2006) observationally derive a primary plateau at $(\mathrm{N} / \mathrm{O})_{\mathrm{PP}}=-1.43$ with objects distributed within a range of -1.54 to -1.27 . In that case, $[\mathrm{N} / \mathrm{O}]_{\mathrm{PP}}=-0.57$. From measurements in blue compact dwarf (BCD) galaxies, Izotov \& Thuan (2004), however, find slightly smaller values with a plateau at about $(\mathrm{N} / \mathrm{O})_{\mathrm{PP}}=-1.6$ or $[\mathrm{N} / \mathrm{O}]_{\mathrm{PP}}=-0.74$.

In Fig. 4 the $[\mathrm{N} / \mathrm{O}]$ abundance ratio is plotted versus the neutral hydrogen column density. It can be seen that there is no 


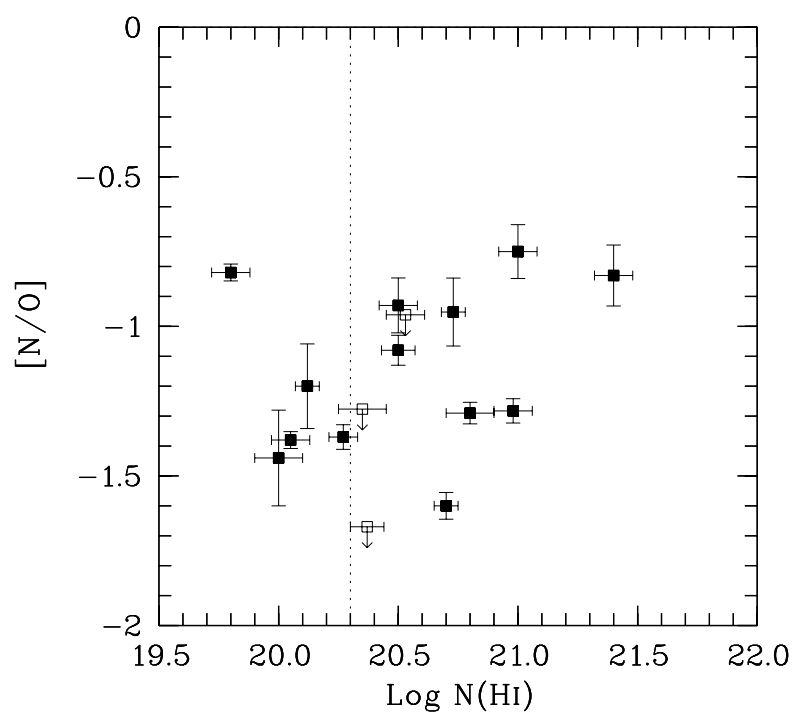

Fig. 4. $[\mathrm{N} / \mathrm{O}]$ abundance ratio versus neutral hydrogen column density $\log N(\mathrm{H} \mathrm{I})$. The vertical dotted line indicates the standard definition of DLA systems $(\log N(\mathrm{H} \mathrm{I})>20.3)$.

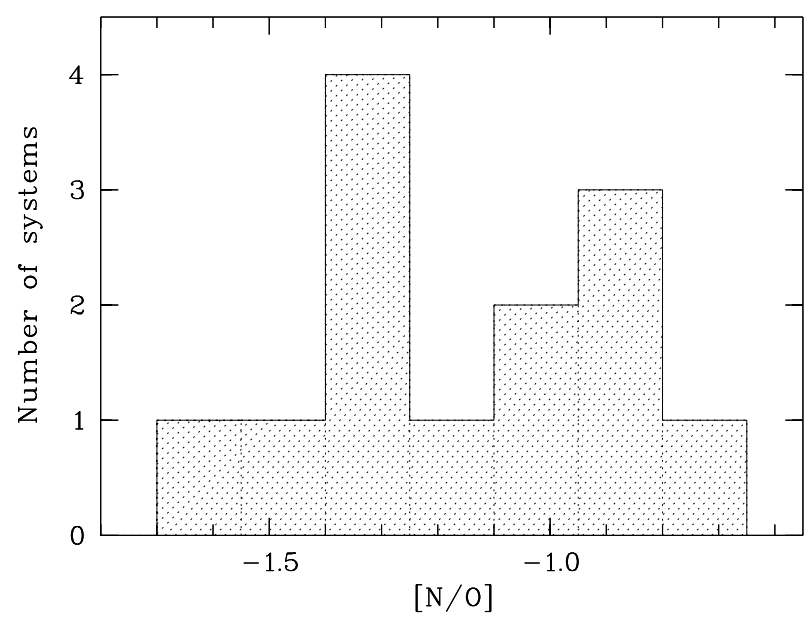

Fig. 5. Histogram of $[\mathrm{N} / \mathrm{O}]$ abundance ratios for the 13 systems with definite measurements in our sample.

apparent correlation between the two parameters. The smallest [N/O] value is found for a system with $\log N(\mathrm{H}$ I $)>20.3$ and one of the largest values is found for the system with the lowest $\log N(\mathrm{H} \mathrm{I})$. This gives additional confidence in the assumption that the ionization correction is negligible when deriving the abundances from $N(\mathrm{O} \mathrm{I}), N(\mathrm{~N} \mathrm{I})$ and $N(\mathrm{H} \mathrm{I})$ (Viegas 1995).

There is a possible dichotomy between systems with $[\mathrm{N} / \mathrm{O}] \sim-0.9$ and -1.4 . It can be seen on Fig. 5 that the distribution of the $[\mathrm{N} / \mathrm{O}]$ abundance ratio has two peaks, one around $[\mathrm{N} / \mathrm{O}] \sim-1.35$ and one around $[\mathrm{N} / \mathrm{O}] \sim-0.9$. This could be, however, a consequence of small number statistics. Out of 13 systems with definite measurements, 7 have $[\mathrm{N} / \mathrm{O}]<-1.15$ and 5 have $[\mathrm{N} / \mathrm{O}]>-1.0$. If we add the two upper limits with $[\mathrm{N} / \mathrm{O}]<-1.2$, we find that the number of systems with $[\mathrm{N} / \mathrm{O}]<-1.15$ is about two times larger than those with $[\mathrm{N} / \mathrm{O}]>-1.0$. A similar dichotomy has already been noted in the $[\mathrm{N} / \alpha]$ ratio by Centurión et al. (2003). They find, however, that $75 \%$ of systems cluster around $[\mathrm{N} / \alpha] \sim-0.87$ and the remaining $25 \%$ cluster around $[\mathrm{N} / \alpha] \sim-1.45$. Also, note that the

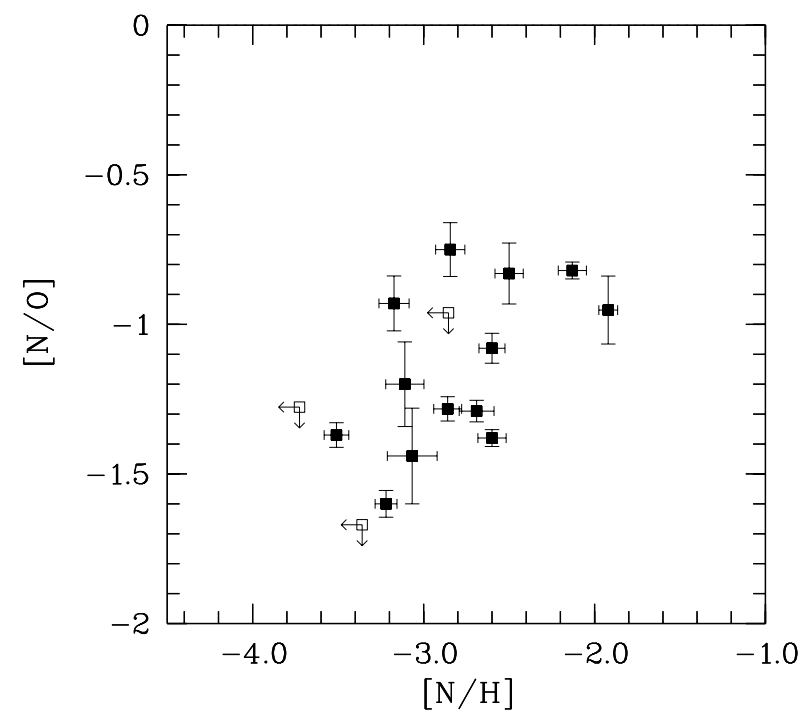

Fig. 6. Nitrogen to oxygen abundance ratio, $[\mathrm{N} / \mathrm{O}]$, versus nitrogen abundance $[\mathrm{N} / \mathrm{H}] .[\mathrm{X} / \mathrm{H}]=(\mathrm{X} / \mathrm{H})-(\mathrm{X} / \mathrm{H})_{\odot}$.

dichotomy present in our data is less pronounced than what is claimed by Centurión et al. (2003) because of a large dispersion of the values below $[\mathrm{N} / \mathrm{O}] \sim-1.2$ (see also Henry \& Prochaska 2007). One could wonder if these differences could be due to our sample being biased against high oxygen metallicity systems compared to the Centurión et al. sample. We do not think this is the case because there is no obvious correlation between $[\mathrm{O} / \mathrm{H}]$ and $[\mathrm{N} / \mathrm{O}]$.

In addition, Centurión et al. (2003) claimed that there is a transition between the two regimes at $[\mathrm{N} / \mathrm{H}] \sim-2.8$ with most, if not all, of the $[\mathrm{N} / \alpha]<-1.5$ systems having $[\mathrm{N} / \mathrm{H}]<-2.8$. Molaro et al. (2003) place this transition at $[\mathrm{N} / \mathrm{H}] \sim-3.0$ and argue that this could be understood as the point at which nitrogen production from AGB stars begins to dominate that of massive stars. Although all the points with $[\mathrm{N} / \mathrm{O}] \leq-1.2$ have $[\mathrm{N} / \mathrm{H}]<-2.4$, we find two systems with $[\mathrm{N} / \mathrm{O}]>-1.2$ and $[\mathrm{N} / \mathrm{H}] \sim-3.0$. Again, below $[\mathrm{N} / \mathrm{O}]<-1$, the scatter in the measurements is larger than above this limit. Note, however, that the four points with $[\mathrm{N} / \mathrm{H}]<-3.2$ have $[\mathrm{N} / \mathrm{O}]<-1.4$ so that the figure is not inconsistent with some kind of break below $[\mathrm{N} / \mathrm{H}]=-3.2$. More data are needed below this limit before any firm conclusion can be drawn.

The classical plot giving $[\mathrm{N} / \mathrm{O}]$ versus $[\mathrm{O} / \mathrm{H}]$ is shown in Fig. 7. Several features have to be noted here. First, all the DLA measurements are located in the region delineated by the usual primary and secondary lines. Recall that, by definition, if nitrogen is a primary element, it should be produced at the same time as oxygen and the $[\mathrm{N} / \mathrm{O}]$ ratio should be a constant whatever the oxygen metallicity is. The primary plateau observed locally is derived from measurements of abundances in nearby low-metallicity galaxies: $[\mathrm{N} / \mathrm{O}]_{\mathrm{PP}}$ in the range -0.57 to -0.74 . If nitrogen is a secondary element, its production is favored at large oxygen abundance and the $[\mathrm{N} / \mathrm{O}]$ ratio should increase with increasing oxygen abundance. The fact that all the DLA systems are found in this region may indicate that at least part of the systems are in the transition zone as a consequence of delays in the release of heavy elements from intermediate mass stars. This is what would be expected if during a starburst, at the beginning, high mass stars eject material with low [N/O] ratio and, after some time, intermediate mass stars eject material with higher 
ratio. The pathway of one particular system in the diagram during his lifetime could be a line starting from the left-bottom corner of the figure towards the up-right direction.

Secondly, DLA measurements are all below the local primary plateau $\left([\mathrm{N} / \mathrm{O}]_{\mathrm{PP}} \sim-0.57\right.$ to -0.74$)$. If we believe that the five top-most [N/O] DLA measurements define what can be considered as a plateau, this plateau is at $[\mathrm{N} / \mathrm{O}] \sim-0.9$, therefore at least -0.15 dex below the local primary plateau. Note that this has not been recognized by studies based on $[\mathrm{N} / \mathrm{Si}]$ or $[\mathrm{N} / \mathrm{S}] \mathrm{ra}-$ tios (Centurión et al. 2002; Pettini et al. 2007). We understand this conclusion is based on small number statistics and should be confirmed with more data. One possibility to explain this would be that DLAs reach the primary plateau only for $[\mathrm{O} / \mathrm{H}]>-1$.

In the lower panel of Fig. 7, we plot $(\mathrm{N} / \mathrm{O})$ vs. $(\mathrm{O} / \mathrm{H})+12$ for DLAs (squares) and local measurements (dots and crosses). The local measurements are from dwarf-irregular galaxy HII regions (van Zee \& Haynes 2006), HII regions in spiral galaxies (van Zee et al. 1998), and metal-poor emission line galaxies (Nava et al. 2006; Izotov et al. 2006). The same remark as above is, of course, to be made here. It is apparent that DLA measurements are all below the local measurements. If a plateau is to be seen in DLA measurements, this is at $(\mathrm{N} / \mathrm{O}) \sim-1.75$. Other points are pretty well scattered in the diagram and we find it difficult to confirm the presence of a second plateau in Fig. 7, as claimed by Centurión et al. (2003).

\section{Conclusion}

We have measured the $[\mathrm{O} / \mathrm{Fe}]$ and $[\mathrm{N} / \mathrm{O}]$ abundance ratios in 13 DLA systems in the range $-2.7<[\mathrm{Fe} / \mathrm{H}]<-1$ and added three systems from the literature.

We show that the scatter in the $[\mathrm{O} / \mathrm{Fe}]$ measurements is small, around a mean value of $[\mathrm{O} / \mathrm{Fe}] \sim 0.32 \pm 0.10$. This small scatter is probably evidence for similar nucleosynthetic histories and efficient mixing of the gas. In the same range of metallicity, $[\mathrm{O} / \mathrm{Fe}]$ in metal-poor stars is found to be about 0.7 dex if the measurements are not corrected for $3 \mathrm{D}$ effects and 0.4 dex if corrected (Cayrel et al. 2004).

In the $[\mathrm{N} / \mathrm{O}]$ versus $[\mathrm{O} / \mathrm{H}]$ diagram, we find that about a third of the measurements are located close to but below the so-called local primary plateau. If a plateau is to be defined for DLAs, its position is at $[\mathrm{N} / \mathrm{O}] \sim-0.9$, which is lower than the plateau measured locally in metal-poor dwarf galaxies, $[\mathrm{N} / \mathrm{O}] \sim-0.57$ to -0.74 . All our measurements are located below the local plateau, which is not the case for measurements in metal-poor halo stars (Spite et al. 2005): about half of the stars have [N/O] $>-0.9$. However, this may be due to small number statistics as a quarter of the DLA systems compiled by Henry \& Prochaska (2007) have a $[\mathrm{N} / \mathrm{Si}]$ ratio larger than the value corresponding to the primary plateau for these elements. We find it difficult to confirm the presence of a second plateau at a lower value, as claimed by Centurión et al. (2003), although the [N/O] distribution is probably double peaked around $[\mathrm{N} / \mathrm{O}] \sim-0.9$ and $[\mathrm{N} / \mathrm{O}] \sim-1.35$. This bimodality in the $[\mathrm{N} / \mathrm{O}]$ distribution could also be due to small number statistics although a possible bimodality in the $[\mathrm{N} / \alpha]$ distribution has already been noticed by Prochaska et al. (2002) and Centurión et al. (2003); see also Henry \& Prochaska (2007).

Oxygen is mainly produced in short-lived massive stars and released into the ISM by type II supernovae explosions. Nitrogen is mainly produced in long-lived intermediate mass stars and ejected into the ISM by stellar winds. This implies delays in the ejection of nitrogen into the ISM even if it is primary. This is probably why most of the DLA measurements are below the local primary plateau. We note that the approximately constant
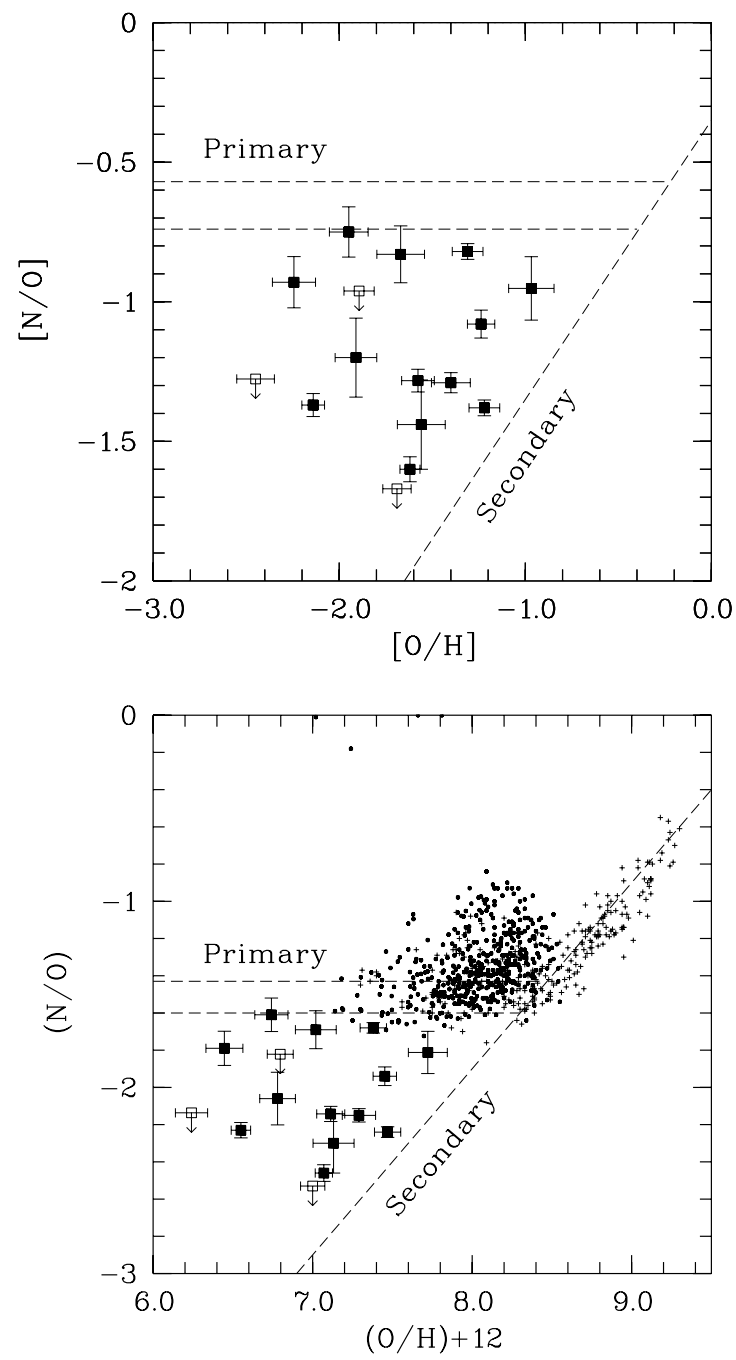

Fig. 7. Upper panel: nitrogen to oxygen abundance ratio, $[\mathrm{N} / \mathrm{O}]$, versus oxygen abundance $[\mathrm{O} / \mathrm{H}] .[\mathrm{X} / \mathrm{H}]=(\mathrm{X} / \mathrm{H})-(\mathrm{X} / \mathrm{H})_{\odot}$. The range for the local primary plateau is indicated as two horizontal dashed lines at $[\mathrm{N} / \mathrm{O}]=-0.57$ and -0.74 . The other dashed line indicates the expected position in case of secondary production: the line is the extrapolation at low $[\mathrm{N} / \mathrm{O}]$ values of local measurements. Lower panel: same as above using the absolute abundances $(\mathrm{X} / \mathrm{H})=\log N(\mathrm{X})-\log N(\mathrm{H})$ with $\mathrm{X}=\mathrm{O}$ or N. The dots and crosses indicate local measurements from dwarfirregular galaxy HII regions (crosses; van Zee \& Haynes 2006), HII regions in spiral galaxies (crosses; van Zee et al. 1998), metal-poor emission line galaxies (dots; Nava et al. 2006), HII regions in BCD galaxies (dots; Izotov \& Thuan 2004) and emission-line galaxies from SDSS (dots; Izotov et al. 2006).

observed $[\mathrm{O} / \mathrm{Fe}]$ ratio does not support the suggestion by Pettini et al. (2002) that iron should have the same evolutionary timescale as nitrogen, thus DLAs deficient in nitrogen should also be deficient in iron. However, their argument holds only for part of the iron production, the part produced by type Ia supernovae that have the same time-scale as intermediate-mass stars. Indeed, models with constant star-formation over a large range of duration produce a $[\mathrm{Si} / \mathrm{Fe}] \sim 0.3$ ratio about constant over a large metallicity range (Henry \& Prochaska 2007). These models cannot reproduce the scatter in the measurements but one could easily claim that constant star-formation rate is a simplistic assumption and that scatter can arise from different star-formation histories (see also Mollá et al. 2006). 

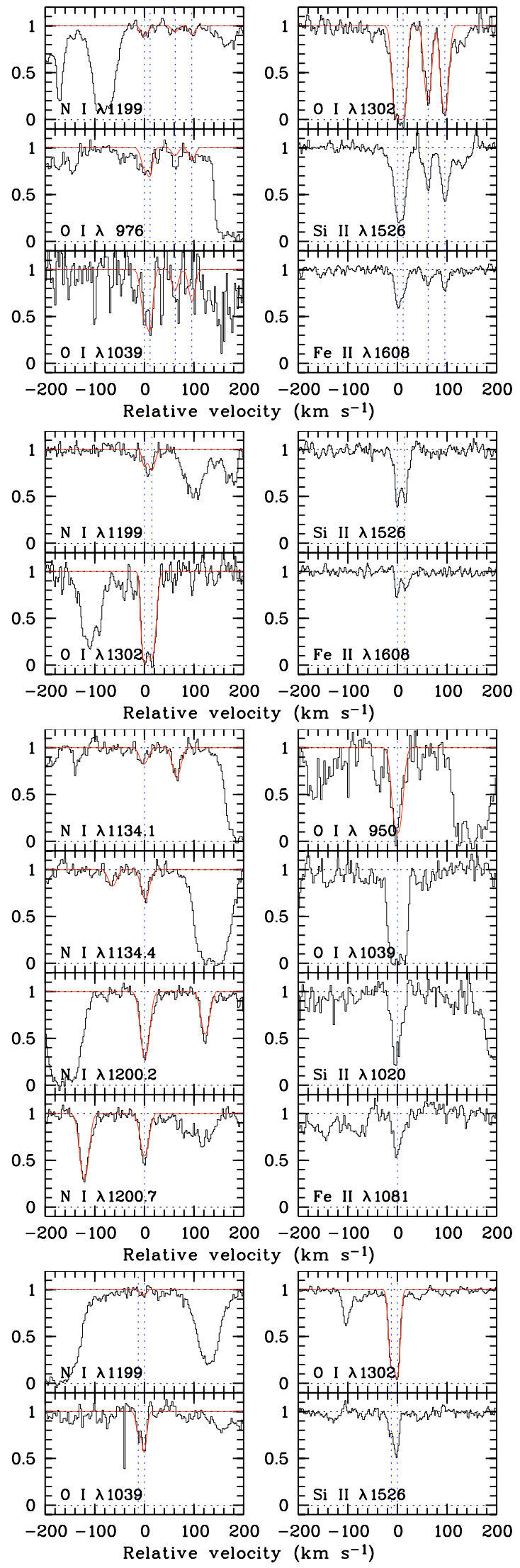

Fig. 8. N I and O I absorption lines in the DLA systems: Q 0102-190, $z=2.926$; Q 0112-306, $z=2.418$; Q 0841+129, $z=2.476$ and Q $0913+072, z=2.618$ from top to bottom, respectively. Model fits are overplotted.
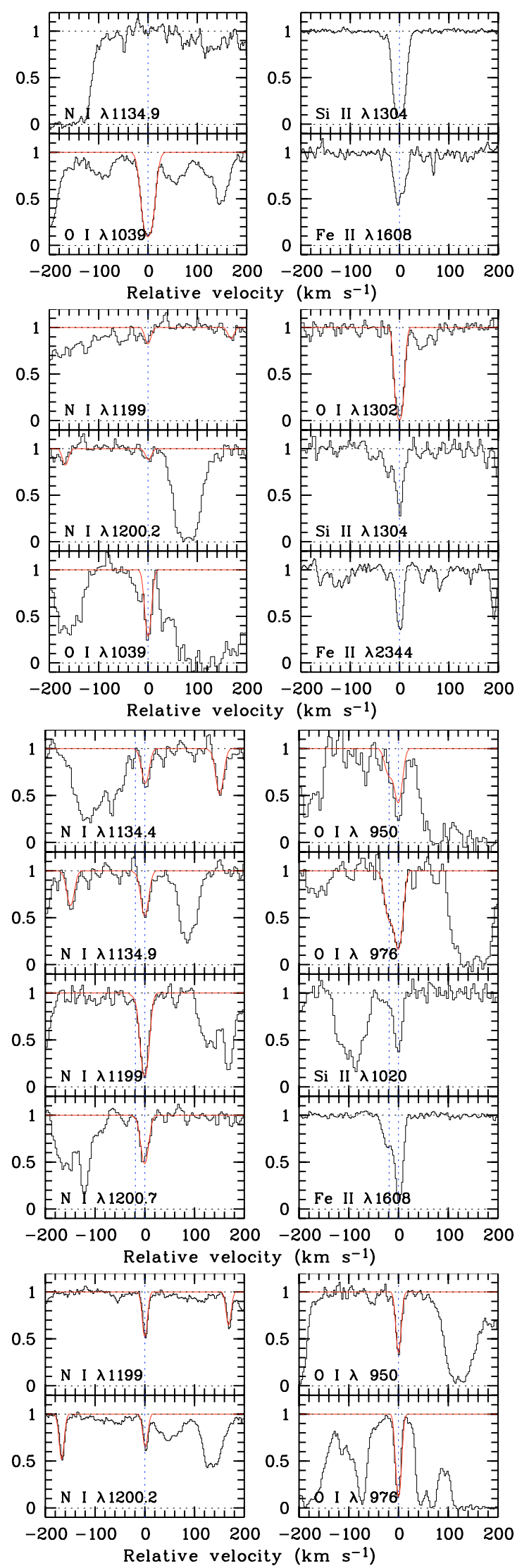

Fig. 9. N I and O I absorption lines in the DLA systems: Q 1108-077, $z=3.608 ; \mathrm{Q} 1337+113, z=2.508$; Q 1337+113, $z=2.796$ and Q $1340-136, z=3.118$ from top to bottom, respectively. Model fits are overplotted. 

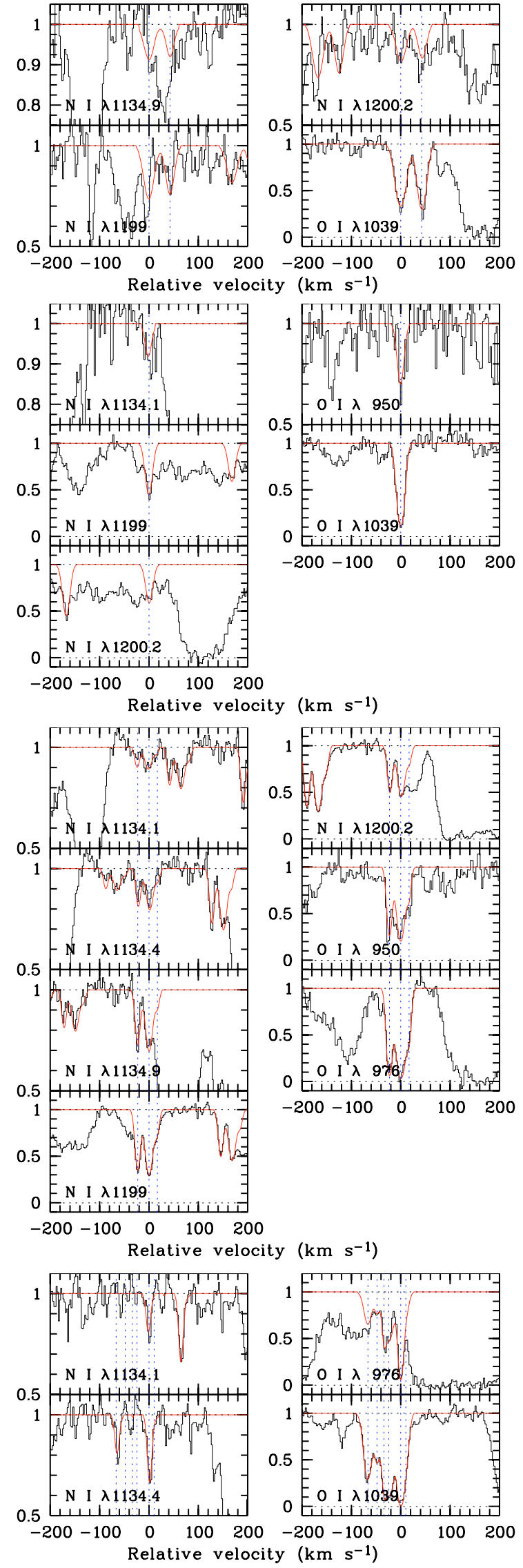

Fig. 10. N I and O I absorption lines in the DLA systems: Q 1409+095, $z=2.456$; Q 1409+095, $z=2.668$; Q 2059-360, $z=3.083$ and Q 2332-094, $z=3.057$ from top to bottom, respectively. Model fits are overplotted.
As a consequence, considering an isolated galaxy, the [N/O] ratio could decrease sharply during a starburst when oxygen is released by $\mathrm{SNe}$ (e.g. Contini et al. 2002). The scatter in the [N/O] ratio measurements could then be due to the intensity of the different bursts of star formation, explaining the different [N/O] values. After some delay, corresponding to the lifetime of intermediate mass stars, the $[\mathrm{N} / \mathrm{O}]$ ratio could increase because of the release of most of the nitrogen. The bimodality of the [N/O] distribution and the larger number of systems with a low [N/O] ratio are a consequence of the delay between the releases of oxygen and nitrogen, because the duration of the release is small compared to the life-time of the stars (see also Ledoux et al. 2006b). It is clear that increasing the sample size would be important to improve our knowledge on these issues.

Acknowledgements. We thank the referee, Paolo Molaro, for a thorough reading of the manuscript and useful comments. R.S. and P.P.J. gratefully acknowledge the Indo-French Centre for the Promotion of Advanced Research (Centre Franco-Indien pour la Promotion de la Recherche Avancée) under contract No. 3004-3. We thank Elisabeth Flam for useful discussions.

\section{References}

Cayrel, R., Depagne, E., Spite, M., et al. 2004, A\&A, 416, 1117 Centurión, M., Molaro, P., Vladilo, G., et al. 2003, A\&A, 403, 55 Contini, T., Treyer, M.A., Sullivan, T., \& Ellis, R.S. 2002, MNRAS, 330, 75 Dessauges-Zavadsky, M., D’Odorico, S., McMahon, R. G., et al. 2001, A\&A, 370,426

Dessauges-Zavadsky, M., Calura, F., Prochaska, J. X., D’Odorico, S., \& Matteucci, F. 2007, A\&A, 470, 431

Erni, P., Richter, P., Ledoux, C., \& Petitjean, P. 2006, A\&A, 451, 19

Grevesse, N., \& Sauval, A. J. 2002, Adv. Spec. Res., 30, 3

Henry, R. B. C., \& Prochaska, J. X. 2007, [arXiv:0708.0236]

Henry, R. B. C., Edmunds, R. G., \& Köppen, J. 2000, ApJ, 541, 660

Izotov, Y. I., \& Thuan, T. X. 1999, ApJ, 511, 639

Izotov, Y. I., \& Thuan, T. X. 2004, ApJ, 602, 200

Izotov, Y. I., Stasińska, G., Meynet, G., Guseva, N. G., \& Thuan, T. X. 2006, A\&A, 448, 955

Knauth, D. C., Meer, D. M., \& Lauroesch, J. T. 2006, ApJ, 647, L115

Ledoux, C., Petitjean, P., \& Srianand, R. 2003, MNRAS, 346, 209

Ledoux, C., Petitjean, P., Fynbo, J.P.U., Möller, P., \& Srianand, R. 2006a, A\&A, 457,71

Ledoux, C., Petitjean, P., \& Srianand, R. 2006b, ApJ, 640, L25

Levshakov, S., Dessauges-Zavadsky, M., D’Odorico, S., \& Molaro, P. 2002, ApJ, 565,696

Lodders, K. 2003, ApJ, 591, 1220

Maeder, A., \& Meynet, G. 2002, A\&A, 390, 561

Marigo, P. 2001, A\&A, 370, 194

Molaro, P., Levshakov, S. A., D’Odorico, S., Bonifacio, P., \& Centurión, M. 2001, ApJ, 549, 90

Molaro, P., Centurión, M., D’Odorico, V., \& Péroux, C. 2003, [arXiv: astro-ph/0307173]

Mollá, M., Vílchez, J. M., \& Díaz, A. I. 2006, MNRAS, 372, 1069

Morton, D. C. 2003, ApJS, 149, 205

Nava, A., Casebeer, D., Henry, R. B. C., \& Jevremovic, D. 2006, ApJ, 645, 1076

Petitjean, P., Bergeron, J., \& Puget, J. L. 1992, A\&A, 265, 375

Petitjean, P., Srianand, R., \& Ledoux, C. 2000, A\&A, 364, L26

Pettini, M., Ellison, S., Bergeron, J., \& Petitjean, P. 2002, A\&A, 391, 21

Pettini, M., Zych, B. J., Steidel, C. C., \& Chaffee, F. H. 2007, [arXiv:0712.1829]

Prochaska, J. X., \& Wolfe, A. M. 2002, ApJ, 566, 68

Prochaska, J. X., \& Wolfe, A. M. 2003, ApJ, 595, 9

Prochaska, J. X., Henry, R. B. C., O’Meara, J. M., et al. 2002, PASP, 114, 933

Spite, M., Cayrel, R., Plez, B., et al. 2005, A\&A, 430, 655

van Zee, J. J., \& Haynes, M. P. 2006, ApJ, 636, 214

van Zee, L., Salzer, J. J., Haynes, M. P., O’Donoghue, A. A. \& Balonek, T. J. 1998, AJ, 116, 2805

Viegas, S. M. 1995, MNRAS, 276, 268

Wolfe, A.M., Lanzetta, K. M., Foltz, C. B., et al., 1995, ApJ, 454, 698

Wolfe, A. M., Gawiser, E., \& Prochaska, J. X. 2005, ARA\&A, 43, 861

Woosley, S. E., \& Weaver, T. A. 1995, ApJS, 101, 181 